Bluttransfusionen bei Hämoglobinopathien

\section{Eisenchelat-Therapie reduziert Morbidität und Mortalität}

Wiederholte Bluttransfusionen, die bei Hämoglobinopathien eine wichtige Behandlungsoption darstellen, können zur Eisenüberladung führen. Eine begleitende Eisenchelat-Therapie, beispielsweise mit Deferasirox, reduziert das Risiko für Organschädigungen. Experten fordern bessere Versorgungsstrukturen, auch weil die Zahl der Betroffenen durch die Flüchtlingskrise deutlich zunimmt.

Bei erblich bedingten Hämoglobinopathien wie Beta-Thalassämie und Sichelzellkrankheit, die vor allem bei Menschen mit Abstammung aus Ländern des Mittelmeerraums, dem Nahen und Mittleren Osten, dem indischen Subkontinent, Asien und Teilen Afrikas gehäuft auftreten, ist die Knochenmarktransplantation (KMT) die einzige kurative Therapie.

Daher wird die Indikation zur allogenen KMT bei der Beta-Thalassämie laut Dr. Stephan Lobitz, Berlin, inzwischen viel häufiger gestellt. Sie ist bei Vorhandensein eines passenden familiären Spenders der Standard und geht mit einem Gesamtüberleben von $97 \%$ einher. In der symptomatischen Therapie spiele Hydroxyurea eine wichtige Rolle, das in Deutschland jedoch erst ab 2 Jahren zugelassen sei, so Lobitz. Eine weitere Option sind Transfusionen. Auch hier werde die Indikation heute häufiger gestellt, erklärte Lobitz. Als letzter Ausweg gilt die haploide KMT.

Bei der Beta-Thalassämie stehen in der symptomatischen Therapie gemäß Dr. Regine Grosse, Hamburg, regelmäßige Transfusionen im Mittelpunkt. Diese werden bei der Thalassaemia major, bei der schwere Verläufe möglich sind, häufig bereits ab den ersten 6 Lebensmonaten vorgenommen. Auch Patienten mit der weniger stark ausgeprägten Thalassaemia intermedia würden heute bereits früher Transfusionen erhalten, erklärte Grosse.

\section{Eisenchelat-Therapie bei Polytransfusionen}

Wie sie fortführte, erfolgen die Transfusionen bei der Beta-Thalassämie in der Regel alle 3-4 Wochen bei einem Hämoglobin (Hb)-Wert von 9,5-10 g/dl, um nicht nur die Anämie zu kompensieren, sondern auch die Eigenerythropoese zu supprimieren. Bereits nach 10-20 Transfusionen besteht allerdings die Gefahr einer Eisenüberladung, weil die Eisenzufuhr die physiologische Eisenausscheidung bei weitem übersteigt. Dadurch reichert sich das Eisen an und kann vielfältige Organschädigungen hervorrufen. Von besonderer Bedeutung seien endokrinologische Funktionsausfälle und Herzschädigungen, so Grosse.

Daher erhalten betroffene Patienten eine Eisenchelat-Therapie, die das überschüssige Eisen über den Urin oder den Stuhl ausschwemmt. $\mathrm{Zu}$ den verfügbaren Chelatoren gehört Deferasirox $\left(\right.$ Exjade $\left.^{\circledR}\right)$, der erste und einzige 1-mal täglich oral einzunehmende Wirkstoff. Grosse empfahl für das Monitoring des Eisens, die Eisenüberladung in der Leber und auch im Pankreas oder Herzen mittels Kernspintomographie zu messen. Nach Daten aus dem Vereinigten Königreich habe sich erst nach Optimierung der Eisenchelat-Therapie im Jahr 1999 die Lebenserwartung sprunghaft verbessert, weil zuvor die meisten Patienten an der Eisenüberladung gestorben seien, hob sie hervor [1].

\section{Versorgungsstruktur verbessern}

Die Zahl der Betroffenen nimmt durch die Flüchtlingskrise nach den Erfahrungen der beiden Experten derzeit in Deutschland deutlich zu. Grosse empfahl, bei einer Schwangeren mit Anämie, die beispielsweise türkische oder arabische Wurzeln hat, nicht nur an einen Eisen- mangel, sondern auch an eine Hämoglobinopathie zu denken. Für die Abklärung, ob der Untersuchte Träger der Krankheitsanlage oder erkrankt ist, gibt es einfache und kostengünstige Verfahren.

Vor diesem Hintergrund forderte Lobitz ein allgemeines Neugeborenen-Screening auf Träger der Mutationen von Hämoglobinopathien, das bereits in vielen europäischen Ländern üblich ist. Darüber hinaus hält er eine Weiterentwicklung der Therapieoptionen und den Aufbau von Expertenzentren für notwendig, damit sowohl Patienten als auch Ärzte schneller kompetente Ansprechpartner finden. Zudem sprach sich Lobitz für ein bundesweites Register aus, das sich für die Sichelzellkrankheit derzeit in der Erprobung befindet, um epidemiologische Daten für Deutschland zu bekommen. Grosse sieht weiteren Optimierungsbedarf bei der Transition, dem Übergang der Versorgung von den Pädiatern zu den Internisten bei zunehmendem Alter der Patienten.

Petra Eiden, Berlin

\section{Quelle}

Presse-Roundtable «Therapie von Hämoglobinopathien - Herausforderungen und Behandlungsstrategien" anlässlich des

5. Hämatologie Heute Symposiums, 20. April 2016, Berlin. Veranstalter: Novartis Oncology

\section{Literatur}

1 Modell B et al.: J Cardiovasc Magn Reson 2008;10:42.

Weitere Informationen bei

Novartis Pharma GmbH

Pia Nothing

pia.nothing@novartis.com

www.novartis.de

www.novartisoncology.de

\title{
Ticker+++ Ticker+++ Ticker+++ Ticker+++ Ticker+++ Ticker+++ Ticker+++
}

Hexal. Im August findet mit dem 26th International Congress of The Transplant Society (TTS) in Hong Kong eine der weltweit größten Veranstaltungen für Transplantationsmediziner statt. Für Fachärzte, die nicht vor Ort sind, stellt Transplant TODAY unter www.transplant-today.com Kongressbeiträge in englischer Sprache zur Verfügung. Experten aus unterschiedlichen europäischen Ländern, darunter 2 renommierte Transplantationsmediziner aus Deutschland, analysieren in Hong Kong die neuesten Studienergebnisse und fassen die Erkenntnisse vor der Kamera zusammen. Die Beiträge können ab dem 21. August, $16 \mathrm{Uhr}$ (CET) ganzjährig abgerufen und als Präsentationsfolien heruntergeladen werden. Der Service Transplant TODAY wird von Sandoz und in Deutschland von Hexal, als Teil der globalen Sandoz-Gruppe, präsentiert.

art tempi communications gmbh

Catrin Schreiner / schreiner@art-tempi.de
CSL Behring. Idelvion ${ }^{\circledR}$ (Albutrepenoncog alfa), das innovative, langwirksame Albumin-Fusionsprotein aus rekombinantem Gerinnungsfaktor IX und rekombinantem Albumin, hat die Zulassung der Europäischen Kommission für die Behandlung und Prophylaxe von Blutungen bei Patienten mit Hämophilie B (kongenitaler Faktor-IX-Mangel) erhalten. Es kann be allen Altersgruppen angewendet werden. Die Zulassung beinhaltet die Langzeitprophylaxe zur Verhinderung oder Verringerung von Blutungsepisoden, die bedarfsorientierte Behandlung sowie das perioperative Blutungsmanagement. Idelvion bietet vorbehandelten Patienten einen hohen Schutz, da bei den meisten Patienten die Faktor-IX-Aktivitätsspiegel nach 14 Tagen noch über 5\% lagen. Als Resultat kann das Intervall zwischen den Infusionen auf 14 Tage ausgeweitet werden.

CSL Behring GmbH www.cslbehring.de 


\section{News}

Transfus Med Hemother 43 | 4 | 16

Neues Hochdurchsatzmodul für die Immunologie von Roche

\section{cobas e 801 - Höchstgeschwindigkeit im Labor}

Nach einem eindrucksvollen Launch-Event im Rahmen der diesjährigen Roche-Tage am 7. Juni 2016 steht Laboren mit dem cobas e 801 ein neues Hochdurchsatzmodul für die Immunologie zur Verfügung. Das einzelne Modul arbeitet mit einem Durchsatz von 300 Tests pro Stunde und wird damit Teil des schnellsten derzeit auf dem Markt verfügbaren immunologischen Analysensystems mit einem Durchsatz von 1200 Tests pro Stunde. Damit unterstützt cobas e 801 Labore in ihrer Produktivität und erlaubt den Anwendern gleichzeitig eine flexible Arbeitsweise: So können unter anderem Reagenzien und Verbrauchsmaterialien jederzeit während der Routine nachgeladen werden.

\section{Produktiv}

Das Modul cobas e 801 bietet maximalen Durchsatz pro Quadratmeter. Das neue Modul für die Testung immunologischer Parameter ermöglicht mit einer Geschwindigkeit von 300 Tests pro Stunde die Durchführung von fast doppelt so vielen Tests wie das aktuelle Modul cobas e 602 . Dabei geht cobas e 801 keine Kompromisse ein und schafft den erhöhten Durchsatz bei gleicher Modulgröße und damit gleichem Platzbedarf, ein Vorteil gerade bei Laboren mit eingeschränkten Platzverhältnissen.

Nicht nur in der Geschwindigkeit setzt cobas e 801 einen neuen Standard, sondern auch in der Anzahl verfügbarer Reagenzkanäle. Mit jeweils 48 Reagenzplätzen pro Modul kann bereits auf 2 Modulen fast das komplette Elecsys-Reagenzportfolio getestet werden, ein Portfolio mit über 100 Parametern.

Schnelligkeit beginnt beim cobas e 801 schon vor der eigentlichen Routine. Durch den neuen Quick-Start-Modus ist das Modul in nur 5,5 Minuten aus dem Standby betriebsbereit. Die hohe Zuverlässigkeit der cobas-Systeme ist wegweisend für die Branche und sorgt für einen reibungslosen Ablauf der Laborroutine und damit für eine unterbrechungsfreie Abarbeitung von Patientenproben.

\section{Flexibel}

Ein weiteres Highlight bei cobas e 801 ist der neu entwickelte Reagenzrotor. Zukünftig können alle Reagenzien und natürlich auch weiterhin sämtliche Verbrauchsmaterialien während der Routine nachgeladen werden. Hierdurch lässt sich die Arbeitsweise mit dem neuen Modul noch besser in den Laboralltag integrieren. Kunden entscheiden selbst, ob sie Reagenzien während der Routine nachladen oder ob durch die flexiblen Packungsgrößen von 100 und 300 Tests pro Packung ein Nachladen überhaupt noch erforderlich ist und sie von den langen Walk-Away-Zeiten profitieren.

Alle Roche-Lösungen zeichnen sich durch ihre maßgeschneiderte Konfiguration aus. Jede Laborlösung ist an die Bedürfnisse des jeweiligen Labors angepasst. Bei Änderungen im Laborablauf lassen sich cobas-Systeme direkt vor Ort erweitern. So können im Labor neue Module an bestehende Analysensysteme angebaut oder auch bestehende Module durch Neuentwicklungen ausgetauscht werden - ein System, das mit den Anforderungen wächst.

\section{Anwenderfreundlich}

Eine weitere Besonderheit des neuen Moduls cobas e 801 ist der gekühlte Reagenzrotor. Der Labormitarbeitende kann die Reagenzien direkt aus dem Kühlschrank auf das Gerät in eine der 48 Reagenzpositionen platzieren, wodurch die Hands-on-Zeit in der Vorbereitung auf die
Routine reduziert wird. Durch die permanente Kühlung der Reagenzien werden Onboard-Stabilitäten von bis zu 120 Tagen auf dem System erreicht. Damit ist die Nutzung von Hochdurchsatz- und Spezialparametern ohne Reagenzverfall möglich.

Auch in punkto Qualität müssen keine Kompromisse eingegangen werden. cobas e 801 nutzt die gleichen Reagenzien wie die aktuellen immunologischen Module von Roche, wodurch sich vergleichbare Wertelagen unabhängig vom verwendeten Analysensystem ergeben.

It's a cobas

Für die Messung der Parameter mit cobas e 801 vertraut Roche weiterhin auf die bewährte Elektrochemilumineszenz-Technologie (ECL), und dies aus gutem Grund. Diese Technologie erlaubt den Anwendern eine schnelle Probenabarbeitung durch kurze Testzeiten, weniger Wiederholungen durch weite Messbereiche und eindeutige Patientenergebnisse durch die hohe Präzision der Technologie. Zur Verdeutlichung ein Beispiel zur Sensitivität von ECL: Heute leben auf der Erde etwa 7,4 Milliarden Menschen. Wenn einer dieser Menschen eine blaue Mütze trüge, wäre ECL in der Lage diese Person zu identifizieren - und das in nur 18 Minuten. Für cobas e 801 wurde die Technologie noch weiter verbessert, sodass in Zukunft mit noch weniger Probenmaterial gearbeitet werden kann. Gerade bei sensiblen Patientengruppen wie Neugeborenen, Krebspatienten, geriatrischen Patienten und Intensivpatienten stellt dies einen wertvollen Beitrag zum "Patient-BloodManagement» dar.

Weitere Informationen bei Roche Diagnostics Deutschland GmbH Sascha Keller sascha_timo.keller@roche.com www.roche.de/diagnostics

\section{Revolade $^{\circledR}$ erhält EU-Zulassung als erster Vertreter einer neuen Wirkstoffklasse zur}

\section{Behandlung von Kindern ab einem Jahr mit chronischer Immunthrombozytopenie}

Die Europäische Kommission hat am 6. April 2016 die Zulassungserweiterung von Revolade ${ }^{\circledR}$ (Eltrombopag) zur Behandlung von Kindern ab einem Jahr mit chronischer Immunthrombozytopenie (cITP) erteilt, die auf vorangegangene Therapien (z.B. Kortikosteroide und Immunglobuline) nicht ausreichend angesprochen haben [1]. Die Zulassung umfasst die Formulierung von Eltrombopag als Tablette sowie als Pulver zur Herstellung einer oral einzunehmenden Suspension, die voraussichtlich ab Herbst 2016 in Deutschland verfügbar sein wird. Eltrombopag ist in der Europäischen Union (EU) seit 2010 zur Behandlung von Erwachsenen mit derselben Indikation zugelassen [1].

Die Zulassungserweiterung basiert auf den Ergebnissen der randomisierten, doppelblinden, placebokontrollierten Studien PETIT* (Phase-IIStudie) und PETIT2, der größten Phase-III-Studie zu dieser Patienten- gruppe [2, 3]. Die Behandlung mit Eltrombopag führte bei therapierefraktären Kindern mit cITP zu einem deutlichen und lang anhaltenden Anstieg der Thrombozytenzahlen [3]. Einige Patienten konnten die Dosis ihrer Begleittherapien (zumeist Kortikosteroide) reduzieren oder diese absetzen [3].

* PETIT = Eltrombopag in PEdiatric patients with Thrombocytopenia from ITP.

Literatur

1 Fachinformation Revolade ${ }^{\circledR}$, Stand Januar 2016.

2 Bussel JB et al.: Lancet Haematol 2015;2:e315-e325.

3 Graigner JD et al.: Lancet 2015;386:1649-1658.

Novartis Pharma GmbH

Pia Nothing / pia.nothing@ novartis.com 


\section{News}

Transfus Med Hemother 43 | 4 | 16

\section{Baxalta unterstützt Forschungsinitiative zur Untersuchung der Therapiebelastung von Patienten mit primärem Immundefekt}

Baxalta Incorporated, ein weltweit agierendes Biopharma-Unternehmen, hat sich der Entwicklung transformativer Therapien für Patienten mit seltenen und bisher wenig beachteten Krankheiten verschrieben. Anlässlich der 6. World Primary Immunodeficiencies Week (WPIW), die vom 22. bis 29. April 2016 stattfand, feierte das Unternehmen seine erzielten Fortschritte bei der Behandlung der primären Immundefekte (PID) und bekräftigte erneut sein Engagement, die globale Gemeinschaft der PID-Patienten zu unterstützen.

Deshalb hat Baxalta ein neues Forschungsvorhaben initiiert. Dieses beschäftigt sich damit, die wichtigsten Einflussfaktoren zu untersuchen, die einen Patienten mit chronischem Immundefekt bei der Bewältigung seiner Er- krankung belasten können. "Baxalta hat sich dazu verpflichtet, das Leben von Patienten mit Immundefekten bedeutsam zu verbessern", sagte Jacopo Leonardi, Executive Vice President und President Immunology von Baxalta. "Dafür ist entscheidend, dass wir die Therapiebelastung von Patienten und auch deren Betreuern besser verstehen. Dies beinhaltet auch die Zeit und den Aufwand, den die Behandlung und das Management der Erkrankung mit sich bringen. Wir unterstützen aktiv die Entwicklung und Verbreitung dieser wichtigen Studie und sind gespannt darauf, wie die Ergebnisse helfen, das Wohlbefinden der PID-Patienten auf der ganzen Welt voranzubringen."

Die gesundheitsökonomische Studie wurde aus einer Partnerschaft zwischen der Universität von Sheffield und der International Patient Organisation for Primary Immunodeficiencies (IPOPI) heraus ins Leben gerufen und wird zusätzlich von der Immune Deficiency Foundation (IDF) in den USA unterstützt. Ziel dieses Programms ist es herauszufinden, welche Belastung bei welcher Therapieoption entsteht und welche Faktoren einen Einfluss auf die Pflegebelastung haben. Die Ergebnisse der Studie sollen dazu dienen, Entscheidungsträger, Ärzte, Gesundheitsdienstleister und Patienten über die Therapiebelastung von PID aufzuklären und ein entsprechendes Bewusstsein $\mathrm{zu}$ schaffen. Außerdem sollen Maßnahmen erkannt werden, die dabei helfen, diese Belastung $\mathrm{zu}$ verringern.

Weitere Informationen bei

Baxalta Incorporated

www.baxalta.com

\section{Studie zu Nplate ${ }^{\circledR}$ zeigt signifikanten Anstieg des dauerhaften Thrombozytenansprechens bei Kindern mit ITP}

Die Ergebnisse einer randomisierten, doppelblinden, placebokontrollierten Phase-III-Studie mit Romiplostim $\left(\right.$ Nplate $^{\circledR}$ ) bei Kindern mit symptomatischer idiopathischer thrombozytopenischer Purpura (Immunthrombozytopenie, ITP) wurden in The Lancet veröffentlicht [1]. Die Studie zeigt, dass 52\% der Patienten unter Romiplostim ein dauerhaftes Thrombozytenansprechen erreichten, verglichen mit 10\% der Patienten im Placebo-Arm ( $\mathrm{p}=0,002$; Odds Ratio (OR) 9,1; 95\%-Konfidenzintervall (KI) 1,9-43,2).

Die Studie belegt, dass mit Romiplostim behandelte Kinder höhere Raten von Gesamtthrombozytenansprechen erlangten. Patienten, die auf Romiplostim ansprachen, wiesen konsistent erhöhte Thrombozytenzahlen auf. Die Befunde zeigen, dass Romiplostim, das gegenwärtig für die Behandlung erwachsener Patienten mit chronischer ITP zugelassen ist, eine mögliche therapeutische Option für Kinder mit symptomatischer ITP mit einer Dauer von 6 oder mehr Monaten ist. Die am häufigsten berichteten unerwünschten Ereignisse schlossen Hämatome, Nasenbluten, Kopfschmerzen und Infektionen der oberen Atemwege ein. Das gesamte Sicherheitsprofil, das im Romiplostim-Arm beobachtet wurde, ähnelte dem bekannten Sicherheitsprofil von Romiplostim.

"Romiplostim unterstützt das Knochenmark dabei, mehr Thrombozyten zu bilden, womit blauen Flecken und Blutungsereignissen vorgebeugt wird. Das ist wichtig für Kinder in dieser Situation. Die Daten fördern das Verständnis, wie Romiplostim eine Rolle dabei spielen könnte, Kindern beim Management dieser Erkrankung zu helfen", so Dr. med. Stefan Kropff,
Medizinischer Direktor bei Amgen Deutschland. «Wir werden mit den Zulassungsbehörden bezüglich einer Zulassung von Romiplostim für pädiatrische Patienten zusammenarbeiten.»

Die Behandlungsziele für Kinder mit ITP sind eine Thrombozytenzahl, die eine angemessene Blutungskontrolle gewährleistet [2], eine Verbesserung der Symptome und eine Steigerung der Thrombozytenzahl $[2,3]$.

\section{Literatur}

1 Tarantino MD et al.: Lancet 2016; doi: 10.1016/S01406736(16)00279-8 (Epub ahead of print).

2 US National Institutes of Health, www.nhlbi.nih.gov/book/ export/html/4917. Letzter Zugriff 22.04.2016.

3 Patient. Trusted medical information and support. http:// patient.info/health/immune-thrombocytopenia-leaflet. Letzter Zugriff: 22.04.2016.

Weitere Informationen bei

Amgen $\mathrm{GmbH}$

www.amgen.de

\section{Ticker+++ Ticker+++ Ticker+++ Ticker+++ Ticker+++ Ticker+++ Ticker+++}

Boehringer Ingelheim initiiert mit RE-SPECT CVT ${ }^{\circledR}$ die 1. prospektive, randomisierte und kontrollierte Studie mit einem nicht-Vitamin-K-antagonistischen oralen Antikoagulans (NOAK) bei Patienten mit Hirn- oder Sinusvenenthrombose. Die Studie untersucht die Sicherheit und Wirksamkeit von Pradaxa ${ }^{\circledR}$ (Dabigatran) bei der Akutbehandlung und Sekundärprävention zerebraler Venenthrombosen (CVT) im Vergleich mit Warfarin. Die neue Studie wurde im Rahmen der 2. Konferenz der European Stroke Organisation in Barcelona, Spanien, vorgestellt. «Wir setzen große Hoffnungen in diese neue Studie. CVT-Patienten brauchen eine effektive Behandlung und wir glauben, dass sie von den jüngsten Fortschritten in der antikoagulatorischen Therapie profitieren können», so Professor José M. Ferro, Lissabon, Portugal.

Boehringer Ingelheim Pharma GmbH \& Co.KG

María Isabel Rodríguez Fernández / presse@ boehringeringelheim.de
Takeda. Adcetris ${ }^{\circledR}$ (Brentuximab Vedotin) hat von der Europäischen Kommission die Zulassung für die konsolidierende Behandlung von erwachsenen Patienten mit CD30+ Hodgkin-Lymphom und erhöhtem Rezidiv- oder Progressionsrisiko nach autologer Stammzelltransplantation (ASCT) erhalten. Eine ASCT ist die Therapie der Wahl bei Patienten, die ein Rezidiv erleiden oder primär therapierefraktär sind. Durchschnittlich $50 \%$ dieser Patienten rezidivieren danach erneut. Die Zulassungsempfehlung basiert auf den positiven Ergebnissen der randomisierten, kontrollierten PhaseIII-Studie AETHERA, die unter der Konsolidierungstherapie mit Brentuximab Vedotin eine statistisch signifikante Verbesserung des progressionsfreien Überlebens gegenüber Placebo gezeigt hat.

Takeda Pharma Vertrieb GmbH \& Co. KG www.takeda-oncology.de 